\title{
Classification of Zintl Ion Clusters Using 4n Series Approach
}

\author{
ENOS MASHEIJA RWANTALE KIREMIRE \\ Department of Chemistry and Biochemistry, University of Namibia, \\ Private Bag 13301, Windhoek, Namibia. \\ ${ }^{*}$ Corresponding author E-mail: kiremire15@yahoo.com \\ http://dx.doi.org/10.13005/ojc/320401
}

(Received: July 07, 2016; Accepted: August 08, 2016)

\begin{abstract}
Zintl ion clusters have the industrial potential for possible applications. A good understanding of such clusters in terms of their bonding, structures and chemical reactivity is extremely important. This paper attempts to categorize and predict the shapes of Zintl clusters of simple to medium nuclearity using the $4 \mathrm{n}$ series method.
\end{abstract}

Keywords: Zintl ion, clusters, series, capping, encapsulated, fragment.

INTRODUCTION

Zintl ion clusters are normally generated by the reactions between alkali and alkaline earth elements in anhydrous liquid ammonia or ethylenediamine ${ }^{1}$. They are found to possess polyhedral geometries like boranes or metal carbonyl clusters and Wade-Mingos rules are utilized to categorize and predict their shapes ${ }^{2-3}$. Zintl phases are currently being investigated for potential industrial applications $\mathbf{s}^{4}$. The recently developed $4 n$ series method has also been found to categorize clusters and predict shapes of small to medium clusters quite successfully ${ }^{5-6}$. However, the method has not been applied to analyze Zintl ion clusters in a systematic manner. The aim of this paper is to categorize and predict the shapes of Zintl clusters using the $4 \mathrm{n}$ series method.

\section{RESULTS AND DISCUSSION}

The background of using the $4 \mathrm{n}$ series method

The $4 n$ series method has been discussed in details in earlier work ${ }^{7-8}$. Nonetheless, a brief outline of the method will be given here as well as some examples illustrating of how it is applied. The $4 \mathrm{n}$ method involves the breaking down the cluster formula into mono-skeletal fragments. If it is a main group fragment, it must comprise of ${ }^{4}$ skeletal valence electrons. A carbon atom $[\mathrm{C}]$ or borane fragment $[\mathrm{BH}]$ exactly fulfill this requirement. Thus,

$1[C] \rightarrow 1[4] \rightarrow 1[4+0]=1[4 n+0](n=1) \rightarrow$ $S=4 n+0(n=1)$.

If we have 2 carbon fragments, $2[\mathrm{C}]$, then the result is doubled as follows: $2[C] \rightarrow 2[4]=2[4+0]=2[4 n+0] \rightarrow S=4 n+0(n=2)$. 
If we have $x[C]$ fragments, then ; $x[C]=$ $\mathrm{x}[4+0] \rightarrow \mathrm{S}=4 \mathrm{n}+0(\mathrm{n}=\mathrm{x})$.

If a fragment has more than 4 valence electrons, then the extra electrons are expressed as a digit after $4 \mathrm{n}$. Let us take the example of a $1[\mathrm{CH}]$ fragment.

$1[\mathrm{CH}] \rightarrow 1[4+1]=1[4 n+1] \rightarrow S=4 n+1(n=1) ;$ the valence electron content of the fragment $\mathrm{V}=4(1)+1=5$

$2[\mathrm{CH}] \rightarrow 2[4+1]=2[4 \mathrm{n}+1] \rightarrow \mathrm{S}=4 \mathrm{n}+2(\mathrm{n}=2) ;$ $\mathrm{V}=4(2)+2=10$, the valence electron content of $2[\mathrm{CH}]$ fragments.

What matters most in the $4 \mathrm{n}$ series method is the skeletal element and the valence electron density around it. For instance, a phosphorus fragment, $1[\mathrm{P}]$ with 5 valence electrons is treated in the same way as a $1[\mathrm{CH}]$ fragment which is isolobal to it.

Hence, $1[\mathrm{P}]=1[5] \rightarrow 1[4+1]$ $=1[4 n+1] \rightarrow 4 n+1(n=1)$; for $2[P]=2[4+1] \rightarrow 2[4 n+1] \rightarrow S$ $=4 n+2(n=2)$. We can use the series formula $S=4 n+2(n=2)$ to derive a corresponding hydrocarbon as in Scheme 1:

The skeletal bonds linking up the two carbon atoms is given by $k=2 n-1$ and since $n=2$, $k=2(2)-1=3$. Since $P_{2}$ belongs to the same series $S=4 n+2(n=2), k=2 n-1=2(2)-1=3$. Hence $P_{2}$ is linked by a triple bond $\mathrm{P} \equiv \mathrm{P}$.

Consider a $\left[\mathrm{CH}_{2}\right]$ fragment. The fragment has 6 valence electron content. These are converted into series as follows:
$1\left[\mathrm{CH}_{2}\right] \rightarrow 1[4+2] \rightarrow \mathrm{S}=4 \mathrm{n}+2(\mathrm{n}=1)$. Let us see how two such fragments can be transmuted into series formula.

$$
2\left[\mathrm{CH}_{2}\right] \rightarrow 2[4+2] \rightarrow 2[4 \mathrm{n}+2] \rightarrow \mathrm{S}=4 \mathrm{n}+4(\mathrm{n}=2) .
$$

The corresponding hydrocarbon $\mathrm{F}_{\mathrm{CH}}=\mathrm{C}_{2} \mathrm{H}_{4}$. We know that $\mathrm{C}_{2} \mathrm{H}_{4}$ has a double bond $(\mathrm{C}=\mathrm{C})$. Again the double bond can be derived from the series $S=4 n+4 ; k=$ $2 n-2=2(2)-2=2$. In general, for series of the formula $S=4 n+q, k=2 n-(q / 2)$.

A sulphur flagment, 1[S] alone also has 6 valence electrons like $1\left[\mathrm{CH}_{2}\right]$. The $4 \mathrm{n}$ series method treats $1[\mathrm{~S}]$ as $\left[\mathrm{CH}_{2}\right]$. Thus, $\left.1[\mathrm{~S}] \mathrm{H}\right) 1\left[\mathrm{CH}_{2}\right]$; the two fragments are isolobal. Hence,

$$
\begin{aligned}
& 1[S] \rightarrow 1[6]=1[4+2] \rightarrow 4 n+2(n=1) . \\
& 2[S] \rightarrow 2[4+2] \rightarrow 2[4 n+2]=4 n+4(n=2) .
\end{aligned}
$$

The electron valence content of $2[S]$ is given by $V=4 n+4=4(2)+4=12$. Also the $k$ value of $S_{2} \rightarrow k$ $=2 n-2=2(2)-2=2$. Hence, according to the series, the $\mathrm{S}_{2}$ molecule is doubly bonded $(\mathrm{S}=\mathrm{S})$ as in $\mathrm{C}_{2} \mathrm{H}_{4}$. In general, for a series formula $S=4 n+q$, the corresponding $k$ value is given by $k=2 n-(q / 2)$.

\section{Categorization of Clusters}

The categorization of clusters is easily done using $4 n$ rather than $14 n$ series. Although this has already been discussed in earlier work, it is being summarized here as many readers are not familiar with the $4 n$ series method of categorizing clusters. The categorization follows the sequence (lower series): $S=4 n+2$ (closo), $4 n+4$ (nido), $4 n+6$ (arachno), $4 n+8$ (hypho) and $4 n+10$ (klapo) and so on. The (higher series) are the capping series namely, $S$ $=4 n+0$ (mono-capped), 4n-2(bi-capped), 4n-4(tricapped), 4n-6(tetra-capped), 4n-8(penta-capped), $4 n-10$ (hexa-capped) and so on. Since the work
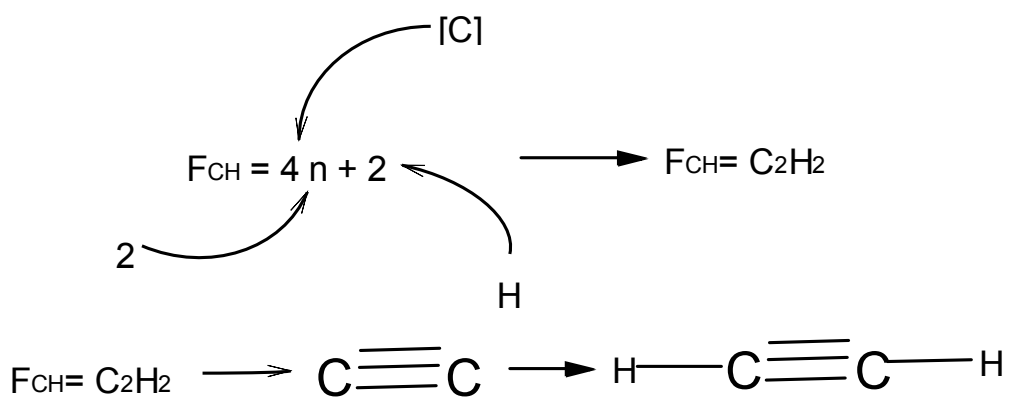

Scheme 1. Converting a series formula into a hydrocarbon 
Table 1: Categorization of Zintl Ion Clusters

\begin{tabular}{|c|c|c|c|c|c|c|}
\hline Cluster & Series & $\mathrm{N}$ Value & Categoryseries & Borane & Hydrocarb & $\mathrm{K}$ Value \\
\hline $\mathrm{Bi}_{2}^{2-}$ & $4 n+4$ & 2 & Nido & $\mathrm{B}_{2} \mathrm{H}_{6}$ & $\mathrm{C}_{2} \mathrm{H}_{4}$ & 2 \\
\hline $\mathrm{Bi}_{3}{ }^{3-}$ & $4 n+6$ & 3 & Arachno & $\mathrm{B}_{3} \mathrm{H}_{9}^{0}$ & $\mathrm{C}_{3} \mathrm{H}_{6}$ & 3 \\
\hline $\mathrm{Sb}_{3}^{3-}$ & $4 n+6$ & 3 & Arachno & $\mathrm{B}_{3} \mathrm{H}_{9}^{9}$ & $\mathrm{C}_{3} \mathrm{H}_{6}^{0}$ & 3 \\
\hline $\mathrm{As}_{3}{ }^{3-}$ & $4 n+6$ & 3 & Arachno & $\mathrm{B}_{3} \mathrm{H}_{9}$ & $\mathrm{C}_{3} \mathrm{H}_{6}^{0}$ & 3 \\
\hline $\mathrm{Ge}_{3}^{4^{-}}$ & $4 n+4$ & 3 & Nido & $\mathrm{B}_{3} \mathrm{H}_{7}$ & $\mathrm{C}_{3} \mathrm{H}_{4}^{0}$ & 4 \\
\hline $\mathrm{Ga}_{3} \mathrm{R}_{3}^{2^{-}}$ & $4 n+2$ & 3 & Closo & $\mathrm{B}_{3} \mathrm{H}_{5}$ & $\mathrm{C}_{3} \mathrm{H}_{2}$ & 5 \\
\hline $\mathrm{Bi}_{4}^{2{ }^{-3}}$ & $4 n+6$ & 4 & Arachno & $\mathrm{B}_{4} \mathrm{H}_{10}^{5}$ & $\mathrm{C}_{4}^{3} \mathrm{H}_{6}^{2}$ & 5 \\
\hline $\mathrm{Pb}_{2} \mathrm{Sb}_{2}{ }^{2^{-}}$ & $4 n+4$ & 4 & Nido & $\mathrm{B}_{4}^{4} \mathrm{H}_{8}$ & $\mathrm{C}_{4}^{4} \mathrm{H}_{4}^{6}$ & 6 \\
\hline $\mathrm{Sn}_{2} \mathrm{Bi}_{2}^{{ }^{2}{ }^{-}}$ & $4 n+4$ & 4 & Nido & $\mathrm{B}_{4}^{4} \mathrm{H}_{8}^{8}$ & $\mathrm{C}_{4}^{4} \mathrm{H}_{4}^{4}$ & 6 \\
\hline $\mathrm{Ge}_{4}^{24^{2}}$ & $4 n+4$ & 4 & Nido & $\mathrm{B}_{4}^{4} \mathrm{H}_{8}^{8}$ & $\mathrm{C}_{4}^{4} \mathrm{H}_{4}^{4}$ & 6 \\
\hline $\mathrm{Pb}_{4}^{4}$ & $4 n+4$ & 4 & Nido & $\mathrm{B}_{4}^{4} \mathrm{H}_{8}^{8}$ & $\mathrm{C}_{4}^{4} \mathrm{H}_{4}^{4}$ & 6 \\
\hline $\mathrm{Sn}_{4}^{4}$ & $4 n+4$ & 4 & Nido & $\mathrm{B}_{4}^{4} \mathrm{H}_{8}^{8}$ & $\mathrm{C}_{4}^{4} \mathrm{H}_{4}^{4}$ & 6 \\
\hline $\ln \mathrm{Bi}_{3}{ }^{2-}$ & $4 n+4$ & 4 & Nido & $\mathrm{B}_{4}^{4} \mathrm{H}_{8}^{8}$ & $\mathrm{C}_{4}^{4} \mathrm{H}_{4}^{4}$ & 6 \\
\hline $\mathrm{Tl}_{2} \mathrm{Te}_{2}^{3}{ }^{2^{-}}$ & $4 n+2$ & 4 & Closo & $\mathrm{B}_{4} \mathrm{H}_{4}^{2-}$ & $\mathrm{C}_{4}^{4} \mathrm{H}_{2}$ & 7 \\
\hline $\mathrm{Al}_{4}^{2^{-2}}$ & $4 n-2$ & 4 & $\mathrm{C}^{2} \mathrm{C}[\mathrm{M}-2]$ & $\mathrm{B}_{4} \mathrm{H}_{2}^{4}$ & $\mathrm{C}_{4}-2 \mathrm{H}^{2}$ & 9 \\
\hline $\mathrm{Bi}_{5}^{4} 5^{-}$ & $4 n+10$ & 5 & Klapo & $\mathrm{B}_{5} \mathrm{H}_{15}$ & $\mathrm{C}_{5} \mathrm{H}_{10}$ & 5 \\
\hline $\mathrm{Bi}_{3} \mathrm{Cr}_{2}(\mathrm{CO})_{6}^{3^{-}}$ & $4 n+2$ & 5 & Closo & $\mathrm{B}_{5} \mathrm{H}_{5} 2^{-2}$ & $\mathrm{C}_{5} \mathrm{H}_{2}$ & 9 \\
\hline $\mathrm{Pb}_{5}^{2^{-2}}$ & $4 n+2$ & 5 & Closo & $\mathrm{B}_{5} \mathrm{H}_{5}{ }^{2-}$ & $\mathrm{C}_{5} \mathrm{H}_{2}^{2}$ & 9 \\
\hline $\mathrm{Sn}_{5}^{5}{ }^{-2}$ & $4 n+2$ & 5 & Closo & $\mathrm{B}_{5} \mathrm{H}_{5}^{5-}$ & $\mathrm{C}_{5}^{5} \mathrm{H}_{2}^{2}$ & 9 \\
\hline $\mathrm{Si}_{4}(\mathrm{CuR})_{2}^{4^{-}}$ & $4 n+0$ & 6 & $\mathrm{C}^{1} \mathrm{C}[\mathrm{M}-5]$ & $\mathrm{B}_{6} \mathrm{H}_{6}$ & $\mathrm{C}_{6}$ & 12 \\
\hline $\mathrm{Bi}_{3} \mathrm{Ni}_{4}(\mathrm{CO})_{6}{ }^{3-}$ & $4 n+2$ & 7 & Closo & $\mathrm{B}_{7} \mathrm{H}_{7}^{2^{-}}$ & $\mathrm{C}_{7} \mathrm{H}_{2}$ & 13 \\
\hline $\mathrm{As}_{7}^{3}$ & $4 n+10$ & 7 & Klapo & $\mathrm{B}_{7} \mathrm{H}_{17}$ & $\mathrm{C}_{7} \mathrm{H}_{10}^{2}$ & 9 \\
\hline $\mathrm{Pb}_{2} \mathrm{Fe}_{3} \mathrm{Cr}_{2}(\mathrm{CO})_{19}{ }^{2-}$ & $4 n+6$ & 7 & Arachno & $\mathrm{B}_{7} \mathrm{H}_{13}$ & $\mathrm{C}_{7} \mathrm{H}_{6}$ & 11 \\
\hline $\mathrm{Pb}_{2} \mathrm{Fe}_{5}(\mathrm{CO})_{17} \mathrm{2}^{-19}$ & $4 n+6$ & 7 & Arachno & $\mathrm{B}_{7} \mathrm{H}_{13}^{13}$ & $\mathrm{C}_{7} \mathrm{H}_{6}^{6}$ & 11 \\
\hline $\mathrm{Pb}_{5} \mathrm{Mo}_{2}(\mathrm{CO})_{6}^{4^{-}}$ & $4 n+0$ & 7 & $\mathrm{C}^{1} \mathrm{C}[\mathrm{M}-6]$ & $\mathrm{B}_{7} \mathrm{H}_{7}$ & $\mathrm{C}_{7}{ }^{6}$ & 14 \\
\hline $\mathrm{Sn}_{8}^{6^{-}}$ & $4 n+6$ & 8 & Arachno & $\mathrm{B}_{8} \mathrm{H}_{14}$ & $\mathrm{C}_{8} \mathrm{H}_{6}$ & 13 \\
\hline $\mathrm{Bi}_{4}^{8} \mathrm{Ni}_{4}(\mathrm{CO})_{6}^{2^{-}}$ & $4 n+2$ & 8 & Closo & $\mathrm{B}_{8} \mathrm{H}_{8}^{2^{-}}$ & $\mathrm{C}_{8} \mathrm{H}_{2}$ & 15 \\
\hline $\mathrm{Si}_{9}^{44^{-4}}$ & $4 n+4$ & 9 & Nido & $\mathrm{B}_{9} \mathrm{H}_{13}$ & $\mathrm{C}_{9} \mathrm{H}_{4}^{2}$ & 16 \\
\hline $\mathrm{Si}_{9}^{3}$ & $4 n+3$ & 9 & Free radical & $\mathrm{B}_{9} \mathrm{H}_{12}$ & $\mathrm{C}_{9} \mathrm{H}_{3}$ & 16.5 \\
\hline $\mathrm{Ge}_{9}^{3-}$ & $4 n+3$ & 9 & Free radical & $\mathrm{B}_{9} \mathrm{H}_{12}$ & $\mathrm{C}_{9} \mathrm{H}_{3}$ & 16.5 \\
\hline $\mathrm{Ge}_{9}^{4^{-}}$ & $4 n+4$ & 9 & Nido & $\mathrm{B}_{9} \mathrm{H}_{13}$ & $\mathrm{C}_{9} \mathrm{H}_{4}^{3}$ & 16 \\
\hline $\mathrm{Sn}_{9}^{4^{-}}$ & $4 n+4$ & 9 & Nido & $\mathrm{B}_{9} \mathrm{H}_{13}$ & $\mathrm{C}_{9} \mathrm{H}_{4}$ & 16 \\
\hline $\ln _{4} \mathrm{Bi}_{5}{ }^{3-}$ & $4 n+4$ & 9 & Nido & $\mathrm{B}_{9} \mathrm{H}_{13}$ & $\mathrm{C}_{9} \mathrm{H}_{4}^{4}$ & 16 \\
\hline $\mathrm{Sn}_{9}^{2^{-5}}$ & $4 n+2$ & 9 & Closo & $\mathrm{B}_{9} \mathrm{H}_{9}{ }^{2^{-}}$ & $\mathrm{C}_{9} \mathrm{H}_{2}^{4}$ & 17 \\
\hline $\mathrm{TISn}_{9}{ }^{3-}$ & $4 n+2$ & 10 & Closo & $\mathrm{B}_{10} \mathrm{H}_{10}^{2}$ & $\mathrm{C}_{10} \mathrm{H}_{2}^{2}$ & 19 \\
\hline $\mathrm{Si}_{9}(\mathrm{ZnPh})^{3^{-}}$ & $4 n+2$ & 10 & Closo & $\mathrm{B}_{10}^{10} \mathrm{H}_{10}^{10} 2^{-}$ & $\mathrm{C}_{10} \mathrm{H}^{1} \mathrm{H}_{2}^{2}$ & 19 \\
\hline $\mathrm{Sn}_{9} \mathrm{Cr}(\mathrm{CO})_{3}{ }^{4-}$ & $4 n+2$ & 10 & Closo & $\mathrm{B}_{10}^{10} \mathrm{H}_{10}^{10} 2^{-}$ & $\mathrm{C}_{10} \mathrm{H}_{2}$ & 19 \\
\hline $\mathrm{Sn}_{9} \mathrm{~W}(\mathrm{CO})_{3}^{4^{-}}$ & $4 n+2$ & 10 & Closo & $\mathrm{B}_{10}^{10} \mathrm{H}_{10}^{10}{ }^{2}$ & $\mathrm{C}_{10} \mathrm{H}_{2} \mathrm{H}_{2}$ & 19 \\
\hline $\mathrm{Pb}_{9} \mathrm{Mo}(\mathrm{CO})_{3}{ }^{4^{-}}$ & $4 n+2$ & 10 & Closo & $\mathrm{B}_{10} \mathrm{H}_{10} \mathrm{2}^{-}$ & $\mathrm{C}_{10} \mathrm{H}_{2} \mathrm{H}_{2}$ & 19 \\
\hline${ }^{*} \mathrm{Sn}_{9} \mathrm{IrL}_{2}{ }^{3^{-}}$ & $4 n+2$ & 10 & Closo & $\mathrm{B}_{10}^{10} \mathrm{H}_{10}^{10} 2^{-}$ & $\mathrm{C}_{10} \mathrm{H}_{2}^{2}$ & 19 \\
\hline $\mathrm{Ge}_{9} \mathrm{Ni}(\mathrm{CO})^{34^{-}}$ & $4 n+1$ & 10 & Free radical & $\mathrm{B}_{10} \mathrm{H}_{11}$ & $\mathrm{C}_{10} \mathrm{H}_{1}$ & 19.5 \\
\hline $\mathrm{Bi}_{3} \mathrm{Ni}_{6}(\mathrm{CO})_{9}{ }^{3-}$ & $4 n+0$ & 9 & $\mathrm{C}^{1} \mathrm{C}[\mathrm{M}-8]$ & $\mathrm{B}_{0} \mathrm{H}_{0}$ & $\mathrm{C}_{\mathrm{o}}$ & 18 \\
\hline${ }^{*} \mathrm{Ge}_{9} \mathrm{CuL}^{3-}$ & $4 n+0$ & 10 & $\mathrm{C}^{1} \mathrm{C}[\mathrm{M}-9]$ & $\mathrm{B}_{10} \mathrm{H}_{10}$ & $C_{10}$ & 20 \\
\hline $\mathrm{Ge}_{9} \mathrm{PdL}^{3^{-}}$ & $4 n-2$ & 10 & $\mathrm{C}^{2} \mathrm{C}[\mathrm{M}-10]$ & $\mathrm{B}_{10} \mathrm{H}_{8}$ & $\mathrm{C}_{10}-2 \mathrm{H}$ & 21 \\
\hline $\ln _{10} Z^{8^{-}}$ & $4 n-4$ & 11 & $\mathrm{C}^{3} \mathrm{C}[\mathrm{M}-8]$ & $\mathrm{B}_{11} \mathrm{H}_{7}$ & $\mathrm{C}_{11}-4 \mathrm{H}$ & 23 \\
\hline
\end{tabular}




\begin{tabular}{|c|c|c|c|c|c|c|}
\hline $\mathrm{TI}_{4} \mathrm{Bi}_{5}{ }^{-3}$ & $4 n+4$ & 9 & Nido & $\mathrm{B}_{9} \mathrm{H}_{13}$ & $\mathrm{C}_{9} \mathrm{H}_{4}$ & 16 \\
\hline $\ln _{5} \mathrm{Bi}_{4}^{5}$ & $4 n+4$ & 9 & Nido & $\mathrm{B}_{9} \mathrm{H}_{13}$ & $\mathrm{C}_{9} \mathrm{H}_{4}$ & 16 \\
\hline $\mathrm{Ge}_{9}^{2^{-2}}$ & $4 n+2$ & 9 & Closo & $\mathrm{B}_{9} \mathrm{H}_{9}^{2-}$ & $\mathrm{C}_{9} \mathrm{H}_{2}$ & 17 \\
\hline $\mathrm{TISn}_{8}{ }^{-3}$ & $4 n+2$ & 9 & Closo & $\mathrm{B}_{9} \mathrm{H}_{9}^{2-}$ & $\mathrm{C}_{9} \mathrm{H}_{2}^{2}$ & 17 \\
\hline $\mathrm{Tl}_{5} \mathrm{Sb}_{4}^{3^{-}}$ & $4 n+2$ & 9 & Closo & $\mathrm{B}_{9} \mathrm{H}_{9}^{2-}$ & $\mathrm{C}_{9} \mathrm{H}_{2}^{2}$ & 17 \\
\hline $\mathrm{Tl}_{6} \mathrm{Sb}_{3}{ }^{-}$ & $4 n+2$ & 9 & Closo & $\mathrm{B}_{9} \mathrm{H}_{9}^{2-}$ & $\mathrm{C}_{9} \mathrm{H}_{2}$ & 17 \\
\hline $\mathrm{Sn}_{6} \mathrm{Cr}_{6}(\mathrm{CO})_{30}{ }^{2^{-}}$ & $4 n+14$ & 12 & - & $\mathrm{B}_{12} \mathrm{H}_{26}$ & $\mathrm{C}_{12} \mathrm{H}_{14}$ & 17 \\
\hline $\mathrm{Sn}_{12}^{12^{-1}}$ & $4 n+12$ & 12 & - & $\mathrm{B}_{12} \mathrm{H}_{24}^{26}$ & $\mathrm{C}_{12} \mathrm{H}_{12}$ & 18 \\
\hline $\mathrm{Ge}_{12}{ }^{2-}$ & $4 n+2$ & 12 & Closo & $\mathrm{B}_{12} \mathrm{H}_{12}^{2}$ & $\mathrm{C}_{12} \mathrm{H}_{2}$ & 23 \\
\hline $\mathrm{TI}_{13}{ }^{11^{-}}$ & $4 n-2$ & 13 & $\mathrm{C}^{2} \mathrm{C}[\mathrm{M}-11]$ & $\mathrm{B}_{13} \mathrm{H}_{11}$ & $\mathrm{C}_{13}-2 \mathrm{H}$ & 27 \\
\hline $\mathrm{Ga}_{6} \mathrm{Sb}_{8}{ }^{14^{-}}$ & $4 n+16$ & 14 & - & $\mathrm{B}_{14} \mathrm{H}_{30}$ & $\mathrm{C}_{14} \mathrm{H}_{16}$ & 20 \\
\hline $\mathrm{ZnAs}_{14}{ }^{4^{-}}$ & $4 n+16$ & 15 & - & $\mathrm{B}_{15}{ }^{14} \mathrm{H}_{31}$ & $\mathrm{C}_{15} \mathrm{H}_{16}$ & 22 \\
\hline $\mathrm{Ni}_{2} \mathrm{Sn}_{17}^{14}$ & $4 n-4$ & 19 & $\mathrm{C}^{3} \mathrm{C}[\mathrm{M}-16]$ & $\mathrm{B}_{19}{ }_{19} \mathrm{H}_{15}^{31}$ & $\mathrm{C}_{19}-4 \mathrm{H}$ & 40 \\
\hline${ }^{*} \mathrm{Ga}_{19} \mathrm{X}_{6}^{-}$ & $4 n-12$ & 19 & $\mathrm{C}^{7} \mathrm{C}[\mathrm{M}-12]$ & $\mathrm{B}_{19} \mathrm{H}_{7}$ & $\mathrm{C}_{19}-12 \mathrm{H}$ & 44 \\
\hline$\left(\mathrm{Si}_{9}\right)_{2}\left[\mathrm{Ni}(\mathrm{CO})_{2}\right]_{2}^{8^{-}}$ & $4 n+8$ & 20 & Hypho & $\mathrm{B}_{20} \mathrm{H}_{28}$ & $\mathrm{C}_{20} \mathrm{H}_{8}$ & 36 \\
\hline $\mathrm{Ga}_{26} \mathrm{G}_{8}{ }^{-2}$ & $4 n-16$ & 26 & $\mathrm{C}^{9} \mathrm{C}[\mathrm{M}-17]$ & $\mathrm{B}_{26} \mathrm{H}_{10}$ & $\mathrm{C}_{26}-16 \mathrm{H}$ & 60 \\
\hline
\end{tabular}

$$
\begin{aligned}
& { }^{*} \mathrm{~L}_{2}=\mathrm{COD},{ }^{* *} \mathrm{~L}=\mathrm{Ph} \\
& { }^{*} \mathrm{X}=\mathrm{CR}_{3}, \mathrm{R}=\mathrm{SiMe}_{3}
\end{aligned}
$$

on $4 \mathrm{n}$ series method is beginning to expand, the terms 'lower series' and 'higher series' are being proposed to refer to non-capping and capping series respectively. It is proposed that the digit after ' $4 n$ ' be referred to as a 'determinant' of the series since it gives an indication of the type of cluster or fragment.

When a transition metal fragment is involved, the relationship $14 n+q H) 4 n+q$ is applied. The $14 n+q$ refers to the transition metal cluster series and $4 n+q$ refers to main group cluster series. It has been found easier to work with $4 n+q$ series rather than $14 n+q$ series. The two series has been found to obey the Hoffmann's isolobal relationship ${ }^{9,5}$. This means that if we have a cluster comprising of skeletal

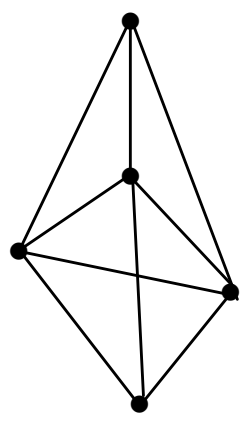

$$
k=9
$$

Trigonal bipyramid, D3h elements from transition and main group elements, the valence electron content of a transition metal are transformed into those of the corresponding main group skeletal element. Let us look at a couple of examples.

$$
\begin{aligned}
& 1\left[\mathrm{Os}(\mathrm{CO})_{3}\right] \rightarrow 1[8+6]=1[14+0] \rightarrow 1[14+0- \\
& 10]=1[4+0] \rightarrow S=4 n+0(n=1) . \\
& 1\left[O s(C O)_{4}\right] \rightarrow 1[8+8]=1[14+2] \rightarrow 1[14+2- \\
& 10]=1[4+2] \rightarrow S=4 n+2(n=1) \\
& 1\left[O s(C O)_{5}\right] \rightarrow 1[8+10]=1[14+4] \rightarrow 1[14+4-10]=1[4+4] \\
& \rightarrow S=4 n+4(n=1)
\end{aligned}
$$

\section{Derivation of Series Formula of Zintl ion Clusters}

$\mathrm{Sn}_{5}{ }^{2-}$

$5[S n] \rightarrow 5[4+0] \rightarrow 4 n+0(n=5)$ $q \rightarrow 0+2(n=0)$

$S=4 n+2(n=5)$, Closo cluster; $k=2 n-1=2(5)-1=9$.

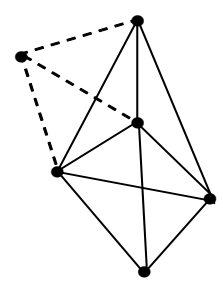

$k=12$

Mono-capped Trigonal bipyramid, D3h 
The equivalent borane cluster, $F_{B}=4 n+2=$ $[\mathrm{BH}](5)+2(-)=\mathrm{B}_{5} \mathrm{H}_{5}{ }^{-}$for closo system. The cluster will be expected to have a similar shape as $\mathrm{B}_{5} \mathrm{H}_{5}{ }^{2}$. The sketch of ideal predicted shape trigonal bipyramid is shown in $\mathrm{F}-1$.

\section{$\mathrm{Bi}_{3} \mathrm{Cr}_{2}(\mathrm{CO})_{6}{ }^{3}$}

$3[\mathrm{Bi}] \rightarrow 3[4+1] \rightarrow 4 \mathrm{n}+3(\mathrm{n}=3)$

$2\left[\mathrm{Cr}(\mathrm{CO})_{4}\right] \rightarrow 2[14+0] \rightarrow 2[14+0-10]=2[4+0] \rightarrow$ $4 \mathrm{n}+0(\mathrm{n}=2)$

$[6-8](\mathrm{CO})=-2(\mathrm{CO}) \rightarrow 0-4(\mathrm{n}=0)$

$\mathrm{q} \rightarrow 0-3(\mathrm{n}=0)$

$S=4 n-4(n=5), C p=C^{3} C[M-2]$; tri-capped cluster.

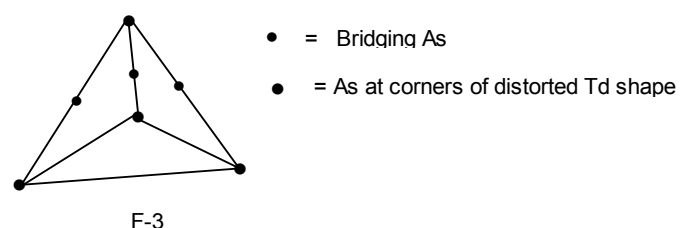

\section{$\mathrm{Si}_{4}(\mathrm{CuR})_{2}{ }^{-}$}

$4[\mathrm{Si}] \rightarrow 4[4+0] \rightarrow 4 \mathrm{n}+0(\mathrm{n}=4)$

$2\left[\mathrm{CuRH}_{2}\right] \rightarrow 4 \mathrm{n}+\mathrm{O}(\mathrm{n}=2)$

$[0-4](\mathrm{H})=-4 \mathrm{H} \rightarrow 0-4(\mathrm{n}=0)$

$\mathrm{q} \rightarrow 0+4(\mathrm{n}=0)$

$S=4 n+0(n=6)$, mono-capped series, $C p=C^{1} C[M-5]$.

This is a mono-capped trigonal bipyramid.

$\mathrm{Pb}_{5} \mathrm{G}_{2}{ }^{-} ; \mathrm{G}=\mathrm{Mo}(\mathrm{CO})_{3}$

$5[\mathrm{~Pb}] \rightarrow 5[4+0] \rightarrow 4 \mathrm{n}+0(\mathrm{n}=5)$

$2\left[\mathrm{Mo}(\mathrm{CO})_{4}\right] \rightarrow 4 \mathrm{n}+0(\mathrm{n}=2)$

$[6-8](C O)=-2(C O) \rightarrow 0-4(n=0)$

$\mathrm{q} \rightarrow 0+4(\mathrm{n}=0)$

$S=4 n+0(n=7) ; C p=C^{\prime} C[M-6]$.

Thus, the series predicts a mono-capped octahedral cluster.

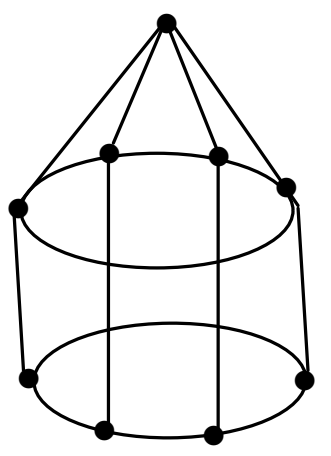

Derivative from $\mathrm{B}_{10} \mathrm{H}_{10} 0^{2-}=\mathrm{B}_{9} \mathrm{H}_{13}$
$\mathrm{As}_{7}{ }^{3-}$

$7[A s] \rightarrow 7[4+1] \rightarrow 4 n+7(n=7)$

$\mathrm{q} \rightarrow 0+3(\mathrm{n}=0)$

$\mathrm{S}=4 \mathrm{n}+10(\mathrm{n}=7) ; \mathrm{k}=2 \mathrm{n}-5=2(7)-5=9$.

A skeletal sketch is shown in F-3A.

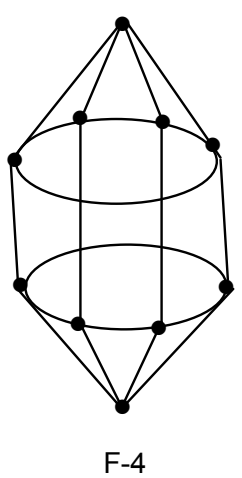

$6 \mathrm{Bi}_{3} \mathrm{Ni}_{4}(\mathrm{CO})_{6}{ }^{3-}$

$3[\mathrm{Bi}] \rightarrow 3[4+1] \rightarrow 4 \mathrm{n}+3(\mathrm{n}=3)$

$4\left[\mathrm{Ni}(\mathrm{CO})_{2}\right] \rightarrow 4 \mathrm{n}+0(\mathrm{n}=4)$

$[6-8](C O)=-2(C O) \rightarrow 0-4(n=0)$

$\mathrm{q} \rightarrow 0+3(\mathrm{n}=0)$

$S=4 n-4(n=7), C p=C^{3} C[M-4]$; tri-capped tetrahedral cluster.

\section{$\mathrm{Bi}_{3} \mathrm{Ni}_{6}(\mathrm{CO})_{9}{ }^{3-}$}

$3[\mathrm{Bi}] \rightarrow 3[4+1] \rightarrow 4 \mathrm{n}+3(\mathrm{n}=3)$

$6\left[\mathrm{Ni}(\mathrm{CO})_{2}\right] \rightarrow 4 \mathrm{n}+0(\mathrm{n}=6)$

$[9-12](\mathrm{CO})=-3(\mathrm{CO}) \rightarrow 0-6(\mathrm{n}=0)$

$\mathrm{q} \rightarrow 0+3(\mathrm{n}=0)$

$S=4 n-6(n=9)(n=9) ; C p=C{ }^{4} C[M-5] ;$ tetra-capped cluster.

$\ln _{4} \mathrm{Bi}_{5}{ }^{3-}$

$4[\ln ] \rightarrow 4[4 n-1]=4 n-4(n=4)$

$5[B i] \rightarrow 5[4+1]=4 n+5(n=5)$

$\mathrm{q} \rightarrow 0+3(\mathrm{n}=0)$

- $=\mathrm{Cr}$

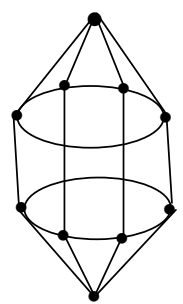

F-5. Sketch of graph representation of $\mathrm{B}_{10} \mathrm{H}_{10} 0^{2-}$ wih one $\mathrm{B}$ replaced by $\mathrm{Cr}$

$S=4 n+4(n=9)$; Nido cluster, $F_{B}=4 n+4=$ $[\mathrm{BH}](9)+4(\mathrm{H})=\mathrm{B}_{9} \mathrm{H}_{13}$. The nido cluster is derived from $\mathrm{B}_{10} \mathrm{H}_{10}{ }^{2-}$ following Rudolph correlation system ${ }^{10}$. This 
is graphically represented in F-3B below.

\section{$\mathrm{Si}_{9}(\mathrm{ZnPh})^{3}$}

$9[\mathrm{Si}] \rightarrow 9[4+0] \rightarrow 4 \mathrm{n}+0(\mathrm{n}=9)$

$1[\mathrm{ZnPh}(\mathrm{H})] \rightarrow 1[14+0] \rightarrow 1[14+0-10]=1[4+0] \rightarrow$ $4 \mathrm{n}+0(\mathrm{n}=1)$

$[0-1](H) \rightarrow 0-1(n=0)$

$q \rightarrow 0+3(n=0)$

$S=4 n+2(n=10)$, closo cluster; $k=2 n-1=$ $2(10)-1=19$.

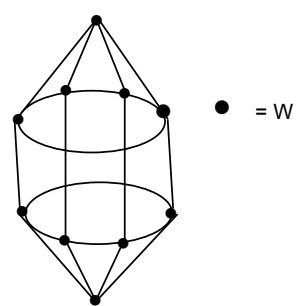

F-6. Sketch of graph representation of $\mathrm{B}_{10} \mathrm{H}_{10}{ }^{2-}$ wih one $\mathrm{B}$ replaced by $\mathrm{W}$

$$
\mathrm{F}_{\mathrm{B}}=4 \mathrm{n}+2=[\mathrm{BH}](10)+2(-1)=\mathrm{B}_{10} \mathrm{H}_{10}{ }^{2-} \text { for }
$$

closo system. The cluster is predicted to have a shape similar to that of $\mathrm{B}_{10} \mathrm{H}_{10}{ }^{2-}$ cluster. This is graphically represented by the skeletal sketch $\mathrm{F}-4$. The shape resembles a beautiful doubly closed basket. One end of the closed tip resides the $\mathrm{Zn}$ skeletal element.

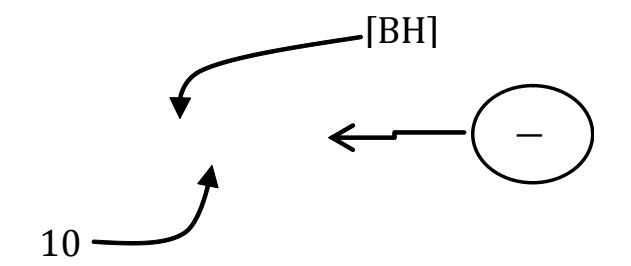

\section{$\mathrm{Sn}_{9} \mathrm{Cr}(\mathrm{CO})_{3}{ }^{-5}$}

$9[\mathrm{Sn}] \rightarrow 9[4+0] \rightarrow 4 \mathrm{n}+0(\mathrm{n}=9)$

$1\left[\mathrm{Cr}(\mathrm{CO})_{4}\right] \rightarrow 4 \mathrm{n}+0(\mathrm{n}=1)$

$[3-4](C O)=-1(C O) \rightarrow 0-2(n=0)$

$\mathrm{q} \rightarrow 0+4(\mathrm{n}=0)$

$S=4 n+2(n=10)$, Closo cluster; $F_{B}=B_{10} H_{10}{ }^{2}$. Cluster expected to have a similar shape as $\mathrm{B}_{10} \mathrm{H}_{10}{ }^{2-}$.

\section{$\mathrm{Sn}_{9} \mathrm{IrL}_{2}{ }^{3}$}

$9[\mathrm{~S} n] \rightarrow 4 n+0(\mathrm{n}=9)$

$1\left[\mathrm{IrHL}_{2}\right] \rightarrow 4 \mathrm{n}+\mathrm{O}(\mathrm{n}=1)$

$[0-1](H)=-1(H) \rightarrow 0-1(n=0)$

$\mathrm{q} \rightarrow 0+3(\mathrm{n}=0)$

$S=4 n+2(n=10)$, Closo

Shape predicted to be similar to that of $\mathrm{B}_{10} \mathrm{H}_{10}{ }^{2-}$.

\section{$\mathrm{Sn}_{9} \mathrm{~W}(\mathrm{CO})_{3}{ }^{-}$}

$9[\mathrm{Sn}] \rightarrow 9[4+0] \rightarrow 4 \mathrm{n}+0(\mathrm{n}=9)$

$1\left[\mathrm{~W}(\mathrm{CO})_{4}\right] \rightarrow 4 \mathrm{n}+0(\mathrm{n}=1)$

$[3-4](C O)=-1(C O) \rightarrow 0-2(n=0)$

$\mathrm{q} \rightarrow 0+4(n=0)$

$\mathrm{S}=4 n+2(n=10)$, Closo cluster; $\mathrm{F}_{B}=\mathrm{B}_{10} \mathrm{H}_{10}{ }^{2-}$. Cluster expected to have a similar shape as $\mathrm{B}_{10} \mathrm{H}_{10}{ }^{2-}$. Isomerism occurs in this case as $\mathrm{W}$ now occupies the equatorial position.

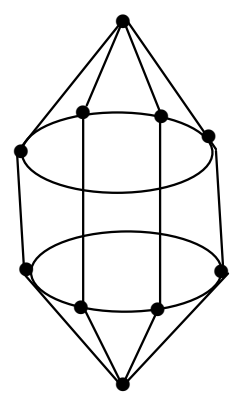

Graph of $\mathrm{Pb}_{10}{ }^{2-}$

$\mathrm{Sn}_{9} \mathrm{IrL}_{2}^{3^{-}}$

$9[S n] \rightarrow 4 n+0(n=9)$

$1\left[\mathrm{IrHL}_{2}\right] \rightarrow 4 \mathrm{n}+0(\mathrm{n}=1)$

$[0-1](H)=-1(H) \rightarrow 0-1(n=0)$

$q \rightarrow 0+3(n=0)$

$S=4 n+2(n=10)$, Closo $^{-}[B H] 10$

Shape predicted to be similar to that of $\mathrm{B}_{10} \mathrm{H}_{10}{ }^{2-}$.

$\mathrm{F}_{\mathrm{B}}=4 \mathrm{n}+2$

\section{$\mathrm{Pb}_{10}{ }^{2}$}

$10[\mathrm{~Pb}] \rightarrow 4 \mathrm{n}+0(\mathrm{n}=10)$

$\mathrm{q} \rightarrow 0+2(\mathrm{n}=0)$

$S=4 n+2(n=10)$, Closo cluster

$\mathrm{F}_{\mathrm{B}}=[\mathrm{BH}](10)+2(-1)=\mathrm{B}_{10} \mathrm{H}_{10}{ }^{2-}$. The negative charge is added since the system is closo. The graphical representation is shown below.

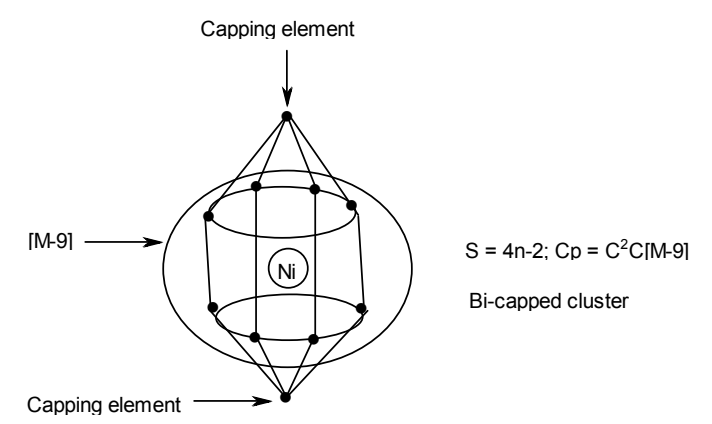

Graph of $\mathrm{Ni@Pb102-}$ 

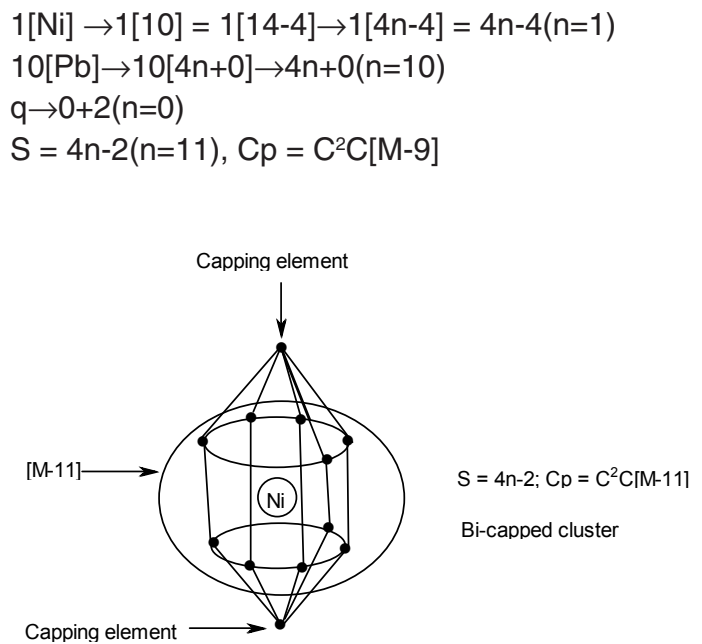

Graph of Ni@Pb12 ${ }^{2-}$

\section{$\mathrm{Ni} @ \mathrm{~Pb}_{12}{ }^{2-}$}

$1[\mathrm{Ni}] \rightarrow 1[10]=1[14-4] \rightarrow 1[4 n-4]=4 n-4(n=1)$

$12[\mathrm{~Pb}] \rightarrow 12[4 \mathrm{n}+0] \rightarrow 4 \mathrm{n}+0(\mathrm{n}=12)$

$\mathrm{q} \rightarrow 0+2(\mathrm{n}=0)$

$S=4 n-2(n=13), C p=C^{2} C[M-11]$.

$$
\mathrm{Cp}=\mathrm{C}^{3} \mathrm{C}[\mathrm{M}-16]
$$

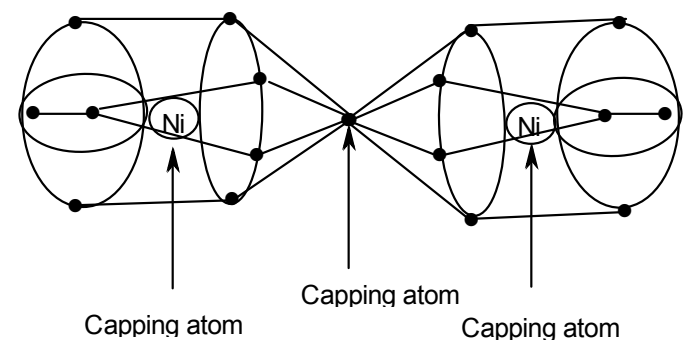

Graph representation of $\mathrm{Ni}_{2} \mathrm{Sn}_{1} 7^{4-}$

This means 2 of the 13 skeletal elements are capped around the remaining 11 others. This could imply the inner set of [5:1:5] skeletal elements as shown in F-9.

$$
\begin{aligned}
& \mathrm{Ni}_{2} \mathrm{Sn}_{17}{ }^{4^{-}} \\
& 2[\mathrm{Ni}] \rightarrow 2[10]=2[14-4] \rightarrow 2[14-4-10]=2[4 n-4] \rightarrow 4 n- \\
& 8(n=2) \\
& 17[\mathrm{Sn}] \rightarrow 17[4+0] \rightarrow 17[4 n+0] \rightarrow 4 n+0(n=17) \\
& q \rightarrow 0+4(n=0) \\
& S=4 n-4(n=19) \rightarrow C p=C^{1}+C^{2} \rightarrow C^{3} C[M-16]
\end{aligned}
$$

According to the $4 n$ series, the cluster is tri-capped. This implies, 3 skeletal atoms are capping versus 16 ones which are not capping. A possible skeletal shape consistent with this prediction in sketched graphically below. Thus, the 2 nickel atoms are regarded as capping in addition to one of the 17 $\mathrm{Sn}$ skeletal elements that acts as a bridge of two $\mathrm{Sn}_{8}$ fragments. This is sketched in F-10.

\section{General considerations}

A wide range of small to medium nulearity Zintl ion clusters taken from several sources ${ }^{11-14}$ have been analyzed and categorized using $4 n$ series method. The results are summarized in Table 1. The common Zintl ion clusters belong to the clusters series $S=4 n+6,4 n+4$ and $4 n+2$. They comprise of skeletal elements ranging from two $(\mathrm{M}-2)$ to thirteen (M-13). As can be seen from Table 1, capping series as defined by $4 \mathrm{n}$ series method are not common. But capping may be induced if a capping fragment is introduced into a cluster. The capping fragment is usually a transition metal atom with ligands or a metal atom or a main group element such as aluminum (Al). A good example of a capping fragment is $\mathrm{M}(\mathrm{CO})_{3}$, $\mathrm{M}=\mathrm{Cr}$, Mo, and $\mathrm{W}$. The valence content $\mathrm{V}$ of the fragment $=12=14-2$. Hence, it belongs to the series $S=14 n-2 H) 4 n-2$ series. If $S_{n}{ }_{9}^{-}$belongs to $S=$ $4 n+4$ (nido) and $M(C O)_{3}$ belongs to $S=4 n-2$ (capping series), then $\mathrm{Sn}_{9}{ }^{-}+\mathrm{M}(\mathrm{CO})_{3 !} \mathrm{Sn}_{9}\left[\mathrm{M}(\mathrm{CO})_{3}\right]^{4^{-}}$and net $S=(4 n+4)+(4 n-2)=4 n+2$ (closo). This means that the nido cluster has been transformed into a closo cluster by adding the capping fragment. The nido cluster $S=4 n+4(n=9)$, corresponds to an equivalent borane $\mathrm{F}_{B}=\mathrm{B}_{9} \mathrm{H}_{13}$ while the closo corresponds to an equivalent closo borane $S=4 n+2(n=10), F_{B}=B_{10} H_{10}{ }^{2}$. The capping fragment, $\mathrm{M}(\mathrm{CO})_{3}$ corresponds to adding $(+\mathrm{B},-\mathrm{H})$ or $[\mathrm{BH}]^{2+}$ fragment to $\mathrm{B}_{9} \mathrm{H}_{13}$ cluster. Other good capping fragments are $\mathrm{Ni}(\mathrm{CO}), \mathrm{S}=$ $4 \mathrm{n}-2$, and $\mathrm{PtL}, \mathrm{L}=\mathrm{PPh}_{3}, \mathrm{~S}=4 \mathrm{n}-2$. Other fragments, $\mathrm{M}(\mathrm{CO})_{2}, \mathrm{M}=\mathrm{Fe}, \mathrm{Ru}$, Os which have a content of 12 valence electrons could be good candidates.

Addition of a metal atom to a closo cluster, may generate a capped cluster. For instance, a nickel atom, [Ni] has 10 valence electrons. According to series it belongs to $14-4$ '!S = $14 n-4 ! 4 n-4$. Therefore it has a capping influence when it is encapsulated in a cluster. For instance, $\mathrm{Pb}_{10}{ }^{2-}(\mathrm{S}=4 \mathrm{n}+2)+\mathrm{Ni}(\mathrm{S}$ $=4 \mathrm{n}-4)^{\prime} ! \mathrm{Ni} @ \mathrm{~Pb}_{10}{ }^{2-}(\mathrm{S}=4 \mathrm{n}-2)$. Thus, the encapsulated cluster becomes a bi-capped cluster, $\mathrm{Cp}=\mathrm{C}^{2} \mathrm{C}[\mathrm{M}-9]$. This implies the [M-9] closo nucleus comprises of 9 atoms arranged as $4: 1: 4$ capped by 2 atoms one 
on one side of the nucleus and the remaining on the other. The whole structure resembles a doubly closed basket as indicated in the sketched graph for 2.2.15. The encapsulated Zintl cluster $\mathrm{Ni} @ \mathrm{~Pb}_{12}{ }^{{ }^{-}}$ can be analyzed in the same way and it is found to belong to the same bi-capped series, $S=4 n-2$ and $\mathrm{Cp}=\mathrm{C}^{2} \mathrm{C}[\mathrm{M}-11]$. This means that 2 skeletal atoms are capping on 11 others. The [M-11] closo atoms are observed to be organized in an approximate order [5:1:5]. The entire doubly closed basket-like closed structure has a skeletal atom arrangement $1:[5: 1: 5]: 1$. This arrangement is shown in a graphical skeletal sketch in 2.2.16 above.

\section{CONCLUSION}

The $4 \mathrm{n}$ series method has been shown to be successful in analyzing, categorizing transition metal carbonyl, boranes, heteroboranes and hydrocarbon clusters. In this paper, it has been demonstrated that it can also readily be applied mainly to the categorization and structural prediction of small to medium size Zintl ion clusters. It is hoped that it may be applied to the analysis and categorization of large Zintl clusters.

\section{ACKNOWLEDGEMENT}

I wish to extend my gratitude to the University of Namibia for providing facilities and financial support. NAMSOV, Namibia is also acknowledged for generous financial support and Mrs Merab Kambamu Kiremire for her continued encouragement to write the articles.

\section{REFERENCES}

1. Scharfe, S., Kraus, F., Stegmaier, S., Fässler, T. F.,Angew., Chem., Int., And., 2011, 50, 3630-3670.

2. Wade, K., J. Chem. Soc., Dalton, 1971, 792793.

3. Mingos, D. M. P., Nature Physical Science, 1972, 236, 99-102.

4. Kauzlarich, S. M., Brown, S. R.,Snyder, G. J., J. Chem. Soc., Dalton, 2007,2099-2107.

5. Kiremire, E. M., Orient. J. Chem., 2015,31(Spl. Edn), 59-70.

6. Kiremire, E. M., Orient. J. Chem., 2015,31(2), 605-618.

7. Kiremire, E. M. R., Int. J. Chem., 2016, 8(1), 145-158.
8. Kiremire, E. M. R., Int. J. Chem., 2016,8(1), 126-144.

9. Hoffmann, R., Angew., Chem., Int., Edn, 1982,21(10),711-800.

10. Rudolph, R.W.,Acc. Chem. Res., 1976,9(12), 446-452.

11. Sevov, S. C., 2008,www3.nd.edu.sevovlab/ articles/c02-6-1. Downloaded 03-07-2016.

12. Goicoechea, J. M., Sevov,S. C., J. Am. Chem. Soc., 2004, 126,6860-6861.

13. Hull, M. W., Sevov, S. C., Angew., Chem., Int. Edn., 2007,46, 6695-6698.

14. Goicoechea, J. M., Sevov,S. C., Organomet, 2006,25,4530-4536. 